Draft version August 11, 2018

Preprint typeset using LATEX style emulateapj v. 2/19/04

\title{
TEMPERATURE INVERSION ON THE SURFACE OF EXTERNALLY HEATED OPTICALLY THICK MULTIGRAIN DUST CLOUDS
}

\author{
DeJAN Vinković \\ Institute for Advanced Study, School of Natural Sciences, Einstein Drive, Princeton, NJ 08540; dejan@ias.edu \\ Draft version August 11, 2018
}

\begin{abstract}
It was recently discovered that the temperature in the surface layer of externally heated optically thick gray dust clouds increases with the optical depth for some distance from the surface, as opposed to the normal decrease in temperature with distance in the rest of the cloud. This temperature inversion is a result of efficient absorption of diffuse flux from the cloud interior by the surface dust exposed to the external radiation. A micron or bigger size grains experience this effect when the external flux is of stellar spectrum. We explore what happens to the effect when dust is a mixture of grain sizes (multigrain). Two possible boundary conditions are considered: i) a constant external flux without constrains on the dust temperature, and ii) the maximum dust temperature set to the sublimation temperature. We find that the first condition allows small grains to completely suppress the temperature inversion of big grains if the overall opacity is dominated by small grains. The second condition enables big grains to maintain the inversion even when they are a minor contributor to the opacity. In reality, the choice of boundary condition depends on the dust dynamics. When applied to the physics of protoplanetary disks, the temperature inversion leads to a previously unrecognized disk structure where optically thin dust can exist inside the dust destruction radius of an optically thick disk. We conclude that the transition between the dusty disk and the gaseous inner clearing is not a sharp edge, but rather a large optically thin region.
\end{abstract}

Subject headings: accretion, accretion disks - circumstellar matter — dust, extinction — stars: pre-main-sequence

\section{INTRODUCTION}

Dust is one of the principal components of interstellar and circumstellar matter. It serves as a very efficient absorber of starlight, which is dominated by visual and ultraviolet photons, and reemiter of this absorbed energy into infrared (IR), thereby modifying the entire spectral energy distribution. Hence, radiative transfer in dusty environments is an inherent part of the study of star formation, protoplanetary disk evolution, dusty winds from AGB stars, circumnuclear environment of AGNs, etc.

In general, radiative transfer is extremely complicated because the dust in these environments always comes as a mixture of various grain sizes (multigrain $\left.{ }^{1}\right)$, shapes and chemical compositions. It is, therefore, common to employ certain approximations that make the problem computationally manageable. For example, dust grains are usually approximated with spheres of similar chemical composition, so that the Mie theory can be easily used for calculating dust cross sections. Another most commonly used approximation is replacing a mixture of dust grain sizes by an equivalent (i.e. synthetic or average) single grain size. Numerical calculations have shown that this approximation does not produce a significant change in the spectral energy distribution (Efstathiou \& Rowan-Robinson 1994; Wolf 2003; Carciofi et al. 2004). It is assumed that this is particularly appropriate for optically thick dust clouds.

It has been discovered recently that externally heated

\footnotetext{
${ }^{1}$ Typically, the term multigrain also includes all other dust grain properties, like the grain shape and chemistry. But, for simplicity, we use this term only to describe grain size effects.
}

optically thick clouds made of large $(\gtrsim 1 \mu \mathrm{m})$ single size dust grains produce the effect of temperature inversion where the maximum temperature is within the dust cloud ${ }^{2}$, at the visual optical depth of $\tau_{V} \sim 1$, instead of on the very surface exposed to the stellar heating (Isella \& Natta 2005; Vinković et al. 2006). The cause of this inversion within the surface layer is its ability to efficiently absorb the diffuse IR radiation originating from the cloud's interior - a process similar to the "greenhouse effect."

It is not known, however, if multigrain dust would produce the same temperature inversion effect. Here we employ analytical multigrain radiative transfer to study the surface of optically thick clouds. Two possible types of boundary conditions are explored: i) a constant external flux heating the cloud, with no limits on the dust temperature, and ii) a fixed maximum dust temperature corresponding to dust sublimation. In $\$ 2$ we describe the analytic method and apply it on single size dust. In the next section $\$ 3$ we apply the method on multigrain dust. After that in 4 we explore a possibility of surface thermal cooling in transverse direction. Some aspects of our result are discussed in $\$ 5$, with our conclusion in $\$ 6$.

\section{OPTICALLY THICK SINGLE SIZE GRAIN DUST CLOUD}

In order to understand effects of multigrain dust on the temperature structure of optically thick clouds, we first have to take a look at the single size dust grain clouds.

2 Another type of temperature inversion has been recognized in protoplanetary disks, where additional viscous heating can increase the disk interior temperature (Calvet et al. 1991; Malbet \& Bertout 1991). In contrast, the temperature inversion discussed here is a pure radiative transfer effect and does not require any additional assumption (like disk viscosity) to operate. 
Here we reiterate analytic techniques described in the literature (Isella \& Natta 2005; Vinković et al. 2006) and then expand our approach to multigrain dust in the following sections.

Consider dust grains at three different locations (Figure 11): on the very surface (point $\mathbf{P}_{0}$ ), at optical depth $\tau_{V}$ (point $\mathbf{P}_{1}$ ) and at optical depth $2 \tau_{V}$ (point $\left.\mathbf{P}_{2}\right)$. The optical depth $\tau_{V}$ is defined at the peak wavelength of the bolometric temperature of external flux $F_{\text {in }}$ illuminating the cloud. We will be interested in cases where dust can reach sublimation temperatures (between $\sim 1,000 \mathrm{~K}$ and $2,000 \mathrm{~K}$ for interstellar dust) and where the external flux source is a star-like object peaking its emission at submicron wavelengths. For the purpose of this paper we use the optical depth $\tau_{V}$ in visual, but one can adjust it to the required wavelength of interest.

The surface layer between points $\mathbf{P}_{0}$ and $\mathbf{P}_{1}$ emits the thermal flux $F_{\text {sur } 1}$. We assume that it emits equally on both sides (toward the left and right in Figure 11). This approximation is valid if the layer is optically thin at the peak wavelength of its IR emission. If we define $q=\sigma_{V} / \sigma_{I R}$, where $\sigma_{V}$ and $\sigma_{I R}$ are dust absorption cross sections in visual and IR, then the IR optical depth requirement is

$$
\tau_{I R}=\frac{\tau_{V}}{q} \lesssim 1
$$

The same requirement holds for the second surface layer between points $\mathbf{P}_{1}$ and $\mathbf{P}_{2}$ and its thermal flux $F_{\text {sur } 2}$. Each of these two layers attenuates the external flux by $\exp \left(-\tau_{V}\right)$ and the thermal fluxes by $\exp \left(-\tau_{I R}\right)=$ $\exp \left(-\tau_{V} / q\right)$.

Since the cloud is optically thick to its own radiation, no net flux can go through the cloud. The total flux coming from the left at any point in the cloud is equal to the total flux coming from the right. This yields flux balance equations at points $\mathbf{P}_{0}, \mathbf{P}_{1}$ and $\mathbf{P}_{2}$

$$
\begin{gathered}
F_{\text {in }}=F_{\text {sur } 1}+F_{\text {sur } 2} e^{-\tau_{V} / q}+F_{\text {out }} e^{-2 \tau_{V} / q} \\
F_{\text {in }} e^{-\tau_{V}}+F_{\text {sur } 1}=F_{\text {sur } 2}+F_{\text {out }} e^{-\tau_{V} / q} \\
F_{\text {in }} e^{-2 \tau_{V}}+F_{\text {sur } 1} e^{-\tau_{V} / q}+F_{\text {sur } 2}=F_{\text {out }}
\end{gathered}
$$

where $F_{\text {out }}$ is the thermal flux coming out of the cloud interior at $\mathbf{P}_{2}$.

We want the external flux $F_{\text {in }}$ at point $\mathbf{P}_{2}$ to be attenuated enough to make diffuse flux the dominant source of heating. This requirement means that $2 \tau_{V} \gtrsim 1$, which in combination with equation 1 gives the allowed range for $\tau_{V}$

$$
0.5 \lesssim \tau_{V} \lesssim q
$$

From equations 2,4 we further get

$$
\begin{aligned}
& F_{\text {sur } 2}=F_{\text {in }} \frac{1+e^{-\tau_{V}}}{1+e^{-\tau_{V} / q}}-F_{\text {out }} e^{-\tau_{V} / q} \\
& F_{\text {sur } 1}=F_{\text {sur } 2}-F_{\text {in }} e^{-\tau_{V}} \frac{1+e^{-\tau_{V}}}{1+e^{-\tau_{V} / q}} .
\end{aligned}
$$

A dust grain at $\mathbf{P}_{0}$ absorbs $\sim \sigma_{V} F_{\text {in }}$ of the external directional flux. It also absorbs $\sim 2 \sigma_{I R} F_{I R}$ of any infrared diffuse flux $F_{I R}$, where the factor 2 accounts for absorption from $2 \pi \mathrm{sr}$. The grain emits at its temperature $T_{0}$ into $4 \pi \mathrm{sr}$, so that the energy balance is

$$
\sigma_{V} F_{\text {in }}+2 \sigma_{I R}\left(F_{\text {sur } 1}+F_{\text {sur } 2} e^{-\tau_{V} / q}+F_{\text {out }} e^{-2 \tau_{V} / q}\right)=
$$

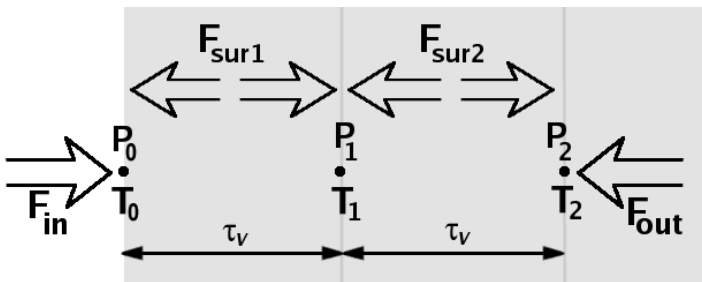

FIG. 1.- Sketch of the surface of an optically thick single size grain dust cloud illuminated from outside. See 2 for details.

$$
=4 \sigma_{I R} \sigma_{S B} T_{0}^{4}
$$

where $\sigma_{S B}$ is the Stefan-Boltzmann constant. Using the flux balance in equation 2 and $q=\sigma_{V} / \sigma_{I R}$, we get

$$
F_{\text {in }}=\frac{4 \sigma_{S B}}{q+2} T_{0}^{4} .
$$

Similarly, we can write the energy balance for a dust grain at $\mathbf{P}_{1}$ and $\mathbf{P}_{2}$

$$
\begin{gathered}
q F_{\text {in }} e^{-\tau_{V}}+2 F_{\text {sur } 1}+2 F_{\text {sur } 2}+2 F_{\text {out }} e^{-\tau_{V} / q}=4 \sigma_{S B} T_{1}^{4} \\
q F_{\text {in }} e^{-2 \tau_{V}}+2 F_{\text {sur } 1} e^{-\tau_{V} / q}+2 F_{\text {sur } 2}+2 F_{\text {out }} . \\
=4 \sigma_{S B} T_{2}^{4}
\end{gathered}
$$

We approximate the interior flux as

$$
F_{\text {out }} \sim \sigma_{S B} T_{2}^{4}
$$

This is a good approximation of the interior for gray dust and an overestimate for non-gray dust, where temperature decreases with optical depth. Now we can continue deriving temperatures in two possible ways.

METHOD 1: Combining equations 617, 9, 10, 11] and 12 yields

$$
\begin{gathered}
T_{1}^{4}=\frac{A+B}{q+2} T_{0}^{4} \\
T_{2}^{4}=\frac{2 B}{q+2} T_{0}^{4} \\
A=q e^{-\tau_{V}}+2\left(1-e^{-\tau_{V}}\right) \frac{1+e^{-\tau_{V}}}{1+e^{-\tau_{V} / q}} \\
B=q e^{-2 \tau_{V}}+2\left(1-e^{-\tau_{V}-\tau_{V} / q}\right) \frac{1+e^{-\tau_{V}}}{1+e^{-\tau_{V} / q}} .
\end{gathered}
$$

The upper panel of Figure 2 shows this temperature profile for $\tau_{V}=1$. The effect of temperature inversion $T_{2}>T_{0}$ is present when $q<q_{\text {limit }} \sim 1$.4. Only grains larger than about one micron can have such a small value of $q$, which makes them almost "gray" in the near IR. This inversion does not appear in non-gray dust $(q>$ $\left.q_{\text {limit }}\right)$ where the temperature decreases monotonically with distance from the cloud surface.

METHOD 2: We can use equations 24 to express $F_{\text {out }}$ as a function of $F_{\text {in }}$ and then use equations 9 and 12 to obtain

$$
T_{2}^{4}=\frac{4}{q+2} \cdot \frac{1+e^{-\tau_{V}}\left(1+e^{-\tau_{V}}-e^{-\tau_{V} / q}\right)}{1+e^{-\tau_{V} / q}} T_{0}^{4}
$$

Similarly, we can express $F_{\text {sur } 1}$ and $F_{\text {sur } 2}$ as a function of $F_{i n}$ and then use equation [10 to obtain

$$
T_{1}^{4}=\frac{1}{q+2}\left[q e^{-\tau_{V}}+2 \frac{2+e^{-\tau_{V}}-e^{-\tau_{V}-\tau_{V} / q}}{1+e^{-\tau_{V} / q}}\right] T_{0}^{4} .
$$




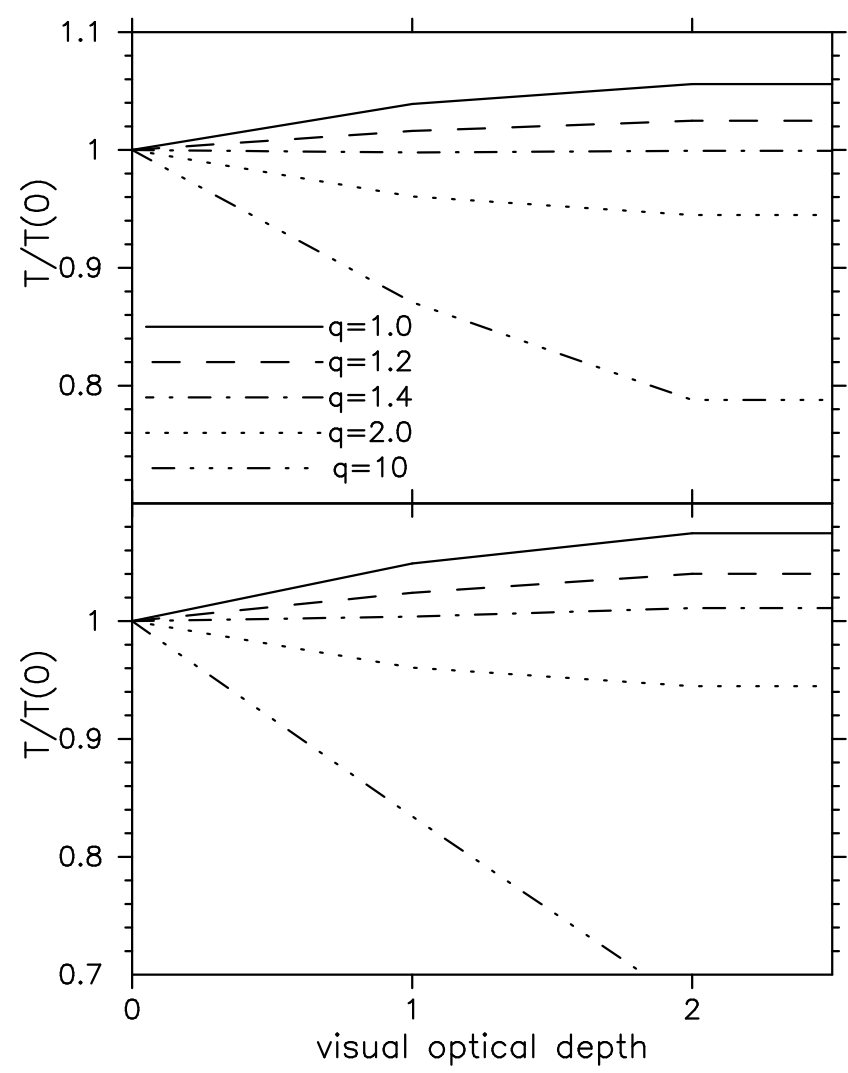

FIG. 2.- Surface temperature of an optically thick single size grain dust cloud for various visual to infrared opacity ratios $q$. The upper panel shows the solution based on equations 13 and 14 (method 1). The lower panel is based on equations [17 and 18 (method 2). Optical depth step is $\tau_{V}=1$. Both solutions show temperature inversion (increase of temperature with optical depth) for small $q$.

The lower panel of Figure 2 shows this result for $\tau_{V}=1$. It differs slightly from previous solution in Method 1, but gives qualitatively the same result. The temperature inversion now exists for $q<q_{\text {limit }} \sim 1.5$. Henceforth, we use Method 1 for further analysis.

\section{OPTICALLY THICK MULTIGRAIN CLOUD}

\subsection{Constant external flux as the boundary condition}

We consider now an externally heated optically thick dust cloud made of $N$ grain sizes, under the condition of constant external flux and no limits on the dust temperature. A dust grain of the $i^{t h}$ size at a point of $\tau_{V}$ optical distance from the cloud surface is heated by the attenuated stellar flux $F_{\text {in }} \exp \left(-\tau_{V}\right)$, by the local diffuse flux $F_{\text {sur }}$ coming from the direction of the surface, and by the local diffuse flux $F_{\text {out }}$ coming out of the cloud interior. The thermodynamic equilibrium of the dust grain gives

$\sigma_{V, i} F_{\text {in }} e^{-\tau_{V}}+2 \sigma_{I R, i}\left(F_{\text {sur }}+F_{\text {out }}\right)=4 \sigma_{I R, i} \sigma_{S B} T_{i}^{4}\left(\tau_{V}\right)$.

Factors 2 and 4 account for absorption from $2 \pi$ sr and emission into $4 \pi$ sr.

On the very surface of the cloud $\tau_{V}=0$ and $F_{\text {sur }}=0$, therefore $F_{\text {in }}=F_{\text {out }}$, which yields

$$
T_{i}^{4}(0)=\left(q_{i}+2\right) \frac{F_{i n}}{4 \sigma_{S B}}
$$

where $q_{i}=\sigma_{V, i} / \sigma_{I R, i}$. This shows that dust grains of different sizes have different temperatures on the surface of an optically thick cloud. The lowest temperature among the grain sizes is acquired by the largest grains because they have the smallest $q_{i}$.

On the other hand, the contribution of the external flux is negligible $F_{\text {in }} \exp \left(-\tau_{V}\right) \rightarrow 0$ when $\tau_{V} \gg 1$, therefore $F_{\text {sur }}=F_{\text {out }}$, which yields the same temperature for all grain sizes

$$
T_{i}^{4}\left(\tau_{V} \gg 1\right)=F_{\text {out }} / \sigma_{S B} .
$$

A general relationship between grain temperatures is derived by subtracting equation 19 for a grain $i$ from the same equation for a grain $j$ and then use equation 20 to obtain

$$
T_{j}^{4}\left(\tau_{V}\right)=T_{i}^{4}\left(\tau_{V}\right)+\frac{q_{j}-q_{i}}{q_{i}+2} e^{-\tau_{V}} T_{i}^{4}(0)
$$

Since $q$ scales inversely with the grain size, equation 22 shows that smaller grains always have a higher temperature than bigger grains at any point in the cloud, with the limit $T_{j} \sim T_{i}$ when $\exp \left(-\tau_{V}\right) \ll 1$.

Optical depth is now a cumulative contribution of all grain sizes in the mix.

$$
\tau_{\lambda} \propto \sum_{i=1}^{N} n_{i} \sigma_{\lambda, i}
$$

where $n_{i}$ is the number density of the $\mathrm{i}^{\text {th }}$ grain size. If we scale optical depth relative to $\tau_{V}$ then

$$
\tau_{I R}=\tau_{V} \frac{\sum_{i=1}^{N} n_{i} \sigma_{I R, i}}{\sum_{i=1}^{N} n_{i} \sigma_{V, i}}=\tau_{V} \sum_{i=1}^{N} \frac{\Upsilon_{V, i}}{q_{i}},
$$

where $\Upsilon_{\lambda, i}$ is the relative contribution of the $i^{\text {th }}$ grain to the dust opacity at wavelength $\lambda$

$$
\Upsilon_{\lambda, i}=\frac{n_{i} \sigma_{\lambda, i}}{\sum_{j=1}^{N} n_{j} \sigma_{\lambda, j}}
$$

Consider now a model similar to figure 1, except that the dust is multigrain. For simplicity and clarity of the following analysis, we work with only two grain sizes: a "big" grain $\alpha$ with $q_{\alpha} \sim 1$ and a "small" grain $\beta$ with $q_{\beta}>q_{\alpha}$. The infrared optical depth step is

$$
\tau_{I R}=\tau_{V}\left(\frac{\Upsilon_{V, \alpha}}{q_{\alpha}}+\frac{\Upsilon_{V, \beta}}{q_{\beta}}\right) .
$$

According to equation 22 the temperature of small grains at optical depth $k \tau_{V}$ (point $\mathbf{P}_{k}$ ) is

$$
T_{k, \beta}^{4}=T_{k, \alpha}^{4}+\frac{q_{\beta}-q_{\alpha}}{q_{\alpha}+2} e^{-k \tau_{V}} T_{0, \alpha}^{4} .
$$

The temperature of big grains at point $\mathbf{P}_{0}$ is (equation 201)

$$
T_{0, \alpha}^{4}=\left(q_{\alpha}+2\right) \frac{F_{i n}}{4 \sigma_{S B}},
$$

while for the other temperatures we need the flux balance at points $\mathbf{P}_{0}, \mathbf{P}_{1}$ and $\mathbf{P}_{2}$. Since the balance is the same as in equations 2,4, except that the infrared step $\tau_{V} / q$ is replaced by $\tau_{I R}$, we use the procedure described in $\$ 2$ and derive

$$
T_{1, \alpha}^{4}=\frac{A_{\alpha}}{q_{\alpha}+2} T_{0, \alpha}^{4}+\frac{F_{\text {out }}}{2 \sigma_{S B}}
$$




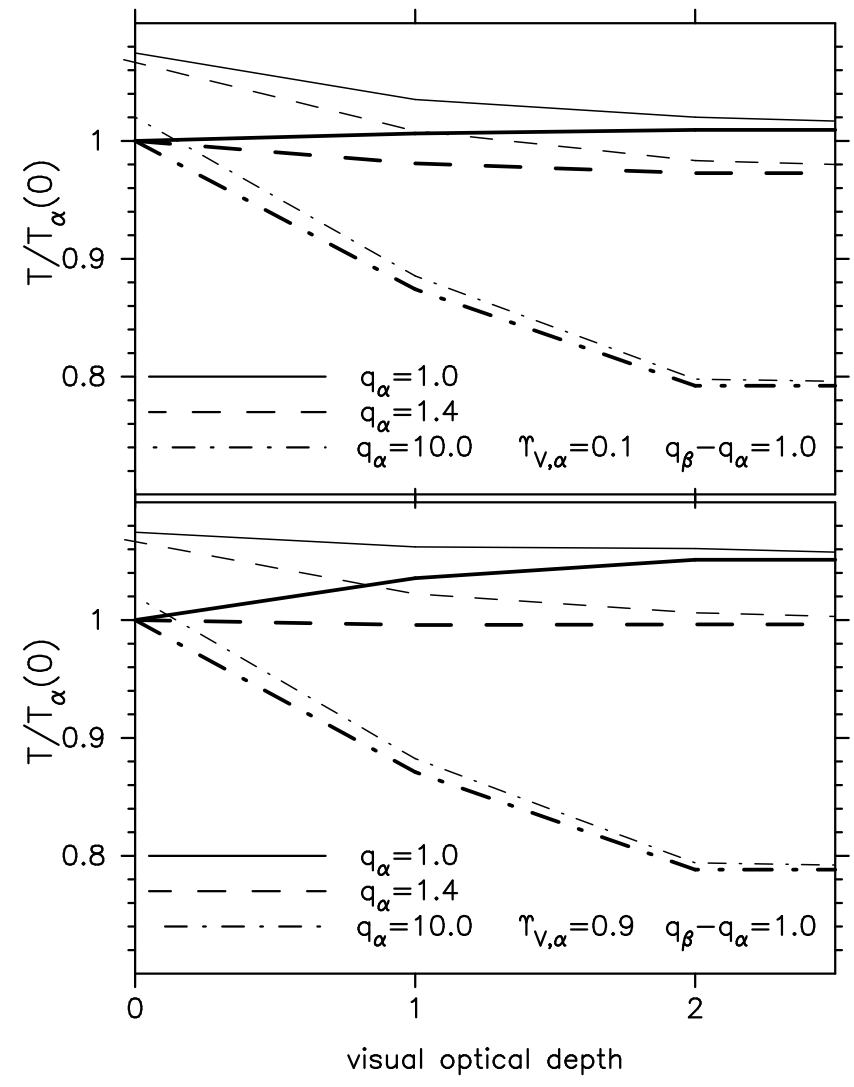

Fig. 3.- Temperature of two types of grains in the surface of an optically thick multigrain dust cloud: larger grains $\alpha$ (thick lines, equations 36 and 37) and smaller grains $\beta$ (thin lines, equation 27). Line styles correspond to different ratios $q_{\alpha}$ of visual to infrared opacity of big grains. The same ratio for small grains is fixed to $q_{\beta}=q_{\alpha}+1$. Panels show results for two relative contributions of big grains to the visual opacity: $\Upsilon_{V \alpha}=0.1$ in the upper panel and $\Upsilon_{V \alpha}=0.9$ in the lower panel. Optical depth step is $\tau_{V}=1$. Notice how the temperature inversion in big grains (small $q_{\alpha}$ ) disappears when small grains dominate the overall opacity (upper panel). The inversion exists only if bigger grains dominate (lower panel).

$$
\begin{gathered}
T_{2, \alpha}^{4}=\frac{B_{\alpha}}{q_{\alpha}+2} T_{0, \alpha}^{4}+\frac{F_{\text {out }}}{2 \sigma_{S B}} \\
A_{\alpha}=q_{\alpha} e^{-\tau_{V}}+2\left(1+e^{-\tau_{V}}\right) \frac{1+e^{-\tau_{V}}}{1+e^{-\tau_{I R}}} \\
B_{\alpha}=q_{\alpha} e^{-2 \tau_{V}}+2\left(1+e^{-\tau_{V}-\tau_{I R}}\right) \frac{1+e^{-\tau_{V}}}{1+e^{-\tau_{I R}}} .
\end{gathered}
$$

The interior flux $F_{\text {out }}$ is now a cumulative contribution of all grain sizes according to their relative contribution to the dust opacity. In our two-size example

$$
F_{\text {out }} / \sigma_{S B}=\Upsilon_{I R, \alpha} T_{2, \alpha}^{4}+\Upsilon_{I R, \beta} T_{2, \beta}^{4} .
$$

Combined with equation 27 gives

$$
\begin{gathered}
F_{\text {out }} / \sigma_{S B}=T_{2, \alpha}^{4}+\frac{C_{\alpha}}{q_{\alpha}+2} T_{0, \alpha}^{4} \\
C_{\alpha}=\Upsilon_{I R, \beta}\left(q_{\beta}-q_{\alpha}\right) e^{-2 \tau_{V}} .
\end{gathered}
$$

Putting together equations 29, 30 and 34 yields the solution

$$
T_{1, \alpha}^{4}=\frac{A_{\alpha}+B_{\alpha}+C_{\alpha}}{q_{\alpha}+2} T_{0, \alpha}^{4}
$$

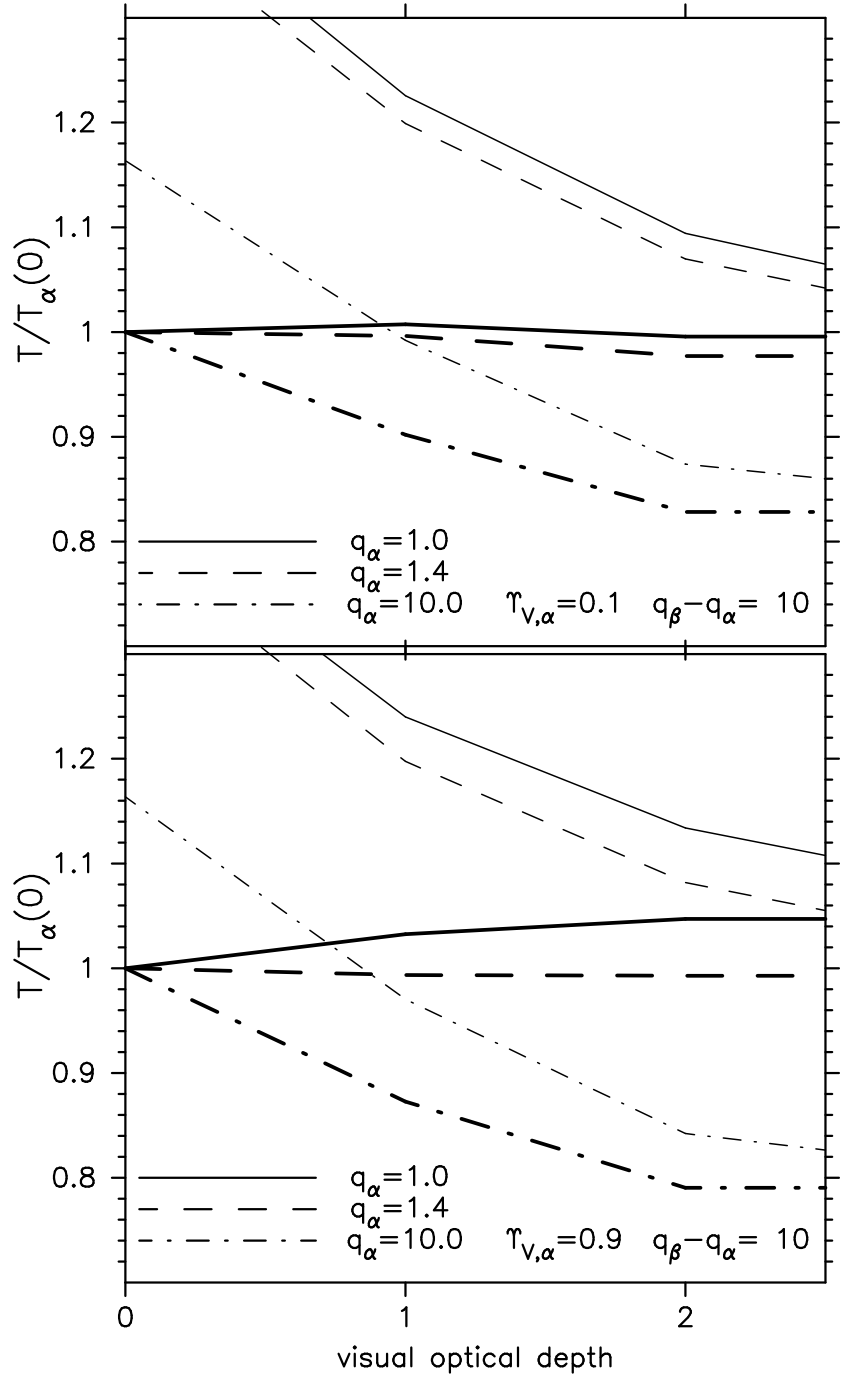

FIg. 4. - Same as Fig. 3 but for $q_{\beta}=q_{\alpha}+10$.

$$
T_{2, \alpha}^{4}=\frac{2 B_{\alpha}+C_{\alpha}}{q_{\alpha}+2} T_{0, \alpha}^{4} .
$$

The resulting temperature is plotted in Figures 3 and 4. The upper panel in each figure shows the result when small grains dominate the opacity $\left(\Upsilon_{V, \alpha}=0.1\right)$. The temperature inversion in big grains is suppressed because the local diffuse flux is dictated by small grains. If big grains dominate the opacity (lower panels, $\Upsilon_{V, \alpha}=0.9$ ) then the temperature inversion is preserved.

\subsection{Sublimation temperature as the boundary condition}

Now we consider a dust cloud hot enough on its illuminated surface to sublimate dust grains warmer than the sublimation temperature $T_{\text {sub }}$. The flux entering the cloud is adjustable to accommodate any temperature boundary condition. From equation 20 we see that small grains are the first to be removed from the immediate surface. If the external flux is high enough then the immediate surface is populated only by $q_{i} \sim 1$ grains ("big grains"). All other grains ("small grains") would survive somewhere within the cloud, at a distance where the local flux is reddened enough by big grains to be absorbed 


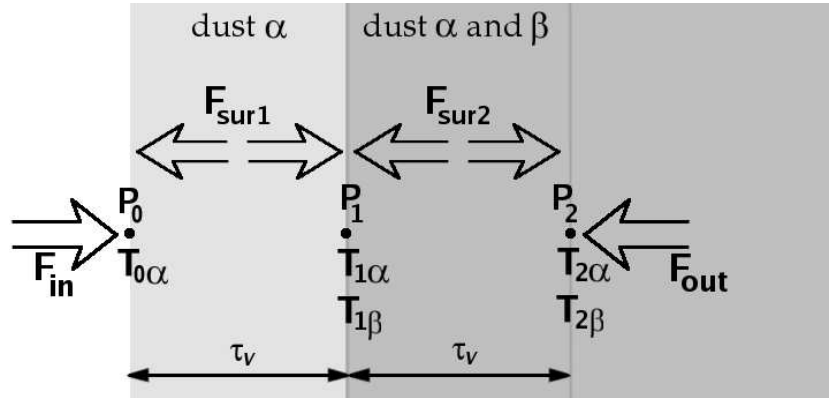

FIG. 5. - Sketch of the surface of an optically thick multigrain dust cloud illuminated from outside. See $\$ 3.2$ for details.

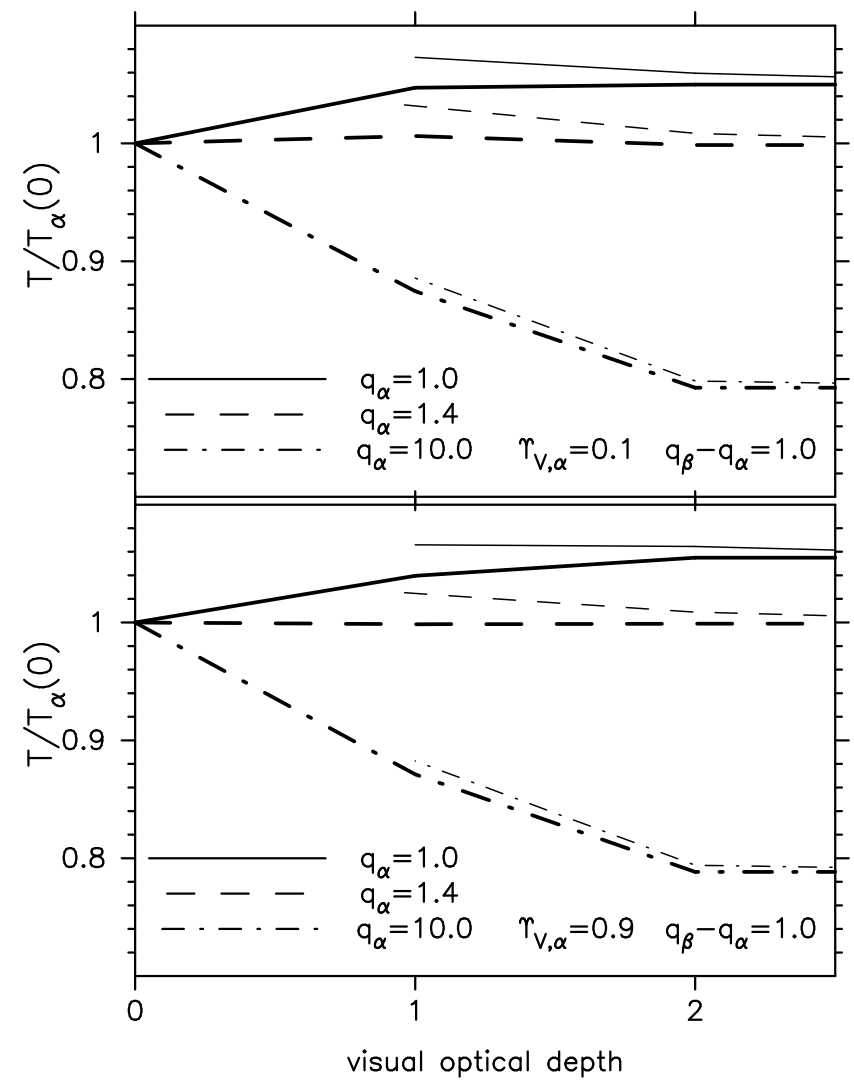

FIG. 6.- Same as Figure 3 except that small grains are removed from the immediate surface by sublimation (Figure 5). The temperature of larger grains is calculated from equations 43 and 44 Despite being mixed with smaller grains, larger grains show temperature inversion even when smaller grains dominate the opacity.

less efficiently.

We again apply our two-size dust model, except that the surface layer of visual optical depth $\tau_{V}$ is occupied only by big grains (see Figure 5). It is too hot for small grains to survive within this layer. Both grains exist at optical distances larger than $\tau_{V}$ from the surface. Following the same procedure as in $\$ 2$, we write the flux balance at points $\mathbf{P}_{0}, \mathbf{P}_{1}$ and $\mathbf{P}_{2}$ (see Figure 5)

$$
\begin{aligned}
F_{\text {in }}= & F_{\text {sur } 1}+F_{\text {sur } 2} e^{-\tau_{V} / q_{\alpha}}+F_{\text {out }} e^{-\tau_{V} / q_{\alpha}-\tau_{I R}} \\
& F_{\text {in }} e^{-\tau_{V}}+F_{\text {sur } 1}=F_{\text {sur } 2}+F_{\text {out }} e^{-\tau_{I R}} \\
& F_{\text {in }} e^{-2 \tau_{V}}+F_{\text {sur } 1} e^{-\tau_{I R}}+F_{\text {sur } 2}=F_{\text {out }}
\end{aligned}
$$

where the IR optical depth between points $\mathbf{P}_{1}$ and $\mathbf{P}_{2}$ is given in equation 26.

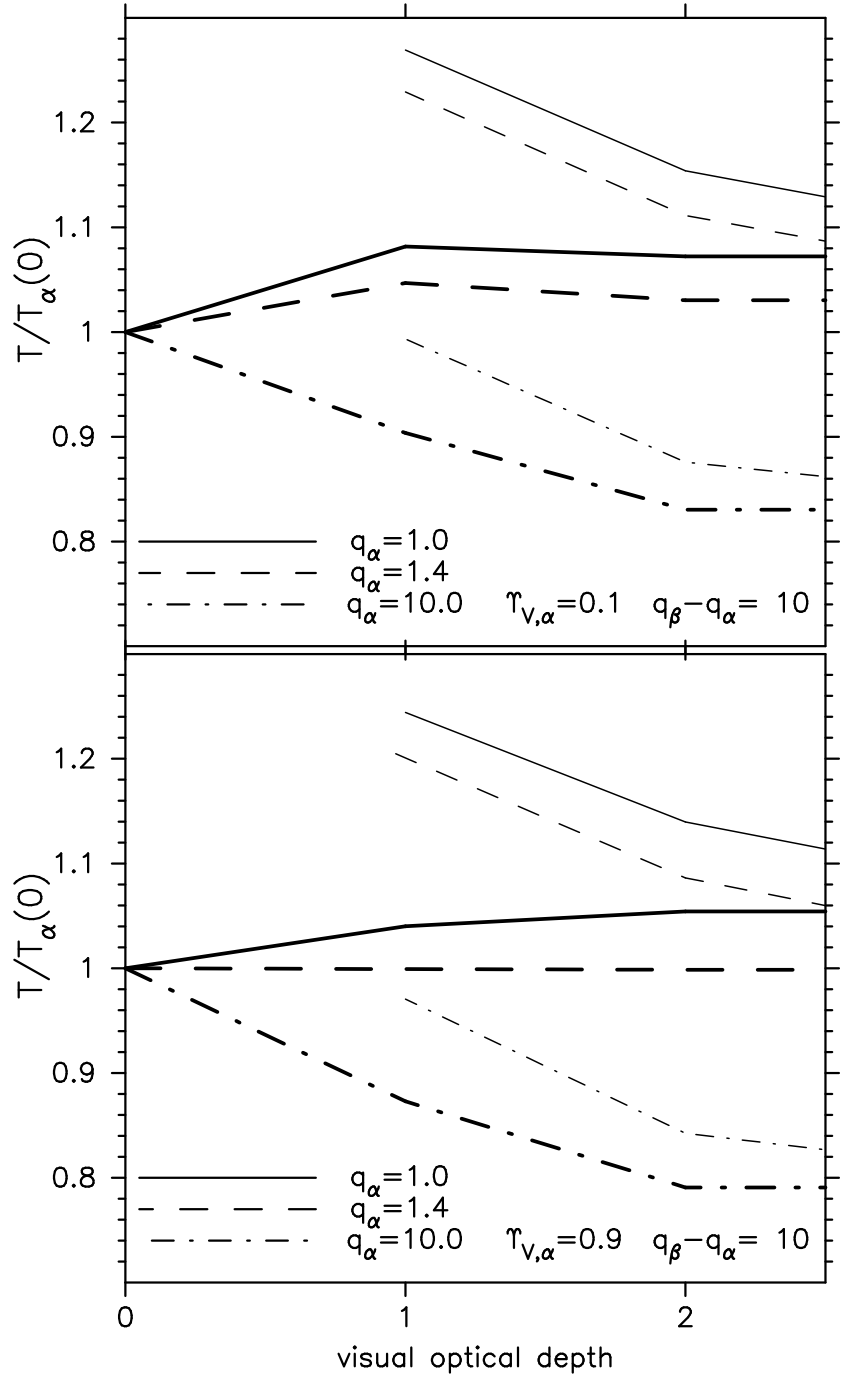

FIG. 7.- Same as Figure 6 but for $q_{\beta}=q_{\alpha}+10$.

Small grains do not exist now at point $\mathbf{P}_{0}$, but their temperature at other points is still described by equation 27. Deriving big grain temperatures at $\mathbf{P}_{1}$ and $\mathbf{P}_{2}$ is now a straightforward procedure already described in $\$ 2$ and 3.1

$$
\begin{gathered}
A_{\alpha}^{\prime}=q_{\alpha} e^{-\tau_{V}}+2 \frac{1+e^{-\tau_{V}}}{1+e^{-\tau_{V} / q_{\alpha}}}-2 e^{-\tau_{V}} \frac{1+e^{-\tau_{V}}}{1+e^{-\tau_{I R}}} . \\
B_{\alpha}^{\prime}=q_{\alpha} e^{-2 \tau_{V}}+2 \frac{1+e^{-\tau_{V}}}{1+e^{-\tau_{V} / q_{\alpha}}}-2 e^{-\tau_{V}-\tau_{I R}} \frac{1+e^{-\tau_{V}}}{1+e^{-\tau_{I R}}} \\
T_{1, \alpha}^{4}=\frac{A_{\alpha}^{\prime}+B_{\alpha}^{\prime}+C_{\alpha}}{q_{\alpha}+2} T_{0, \alpha}^{4} \\
T_{2, \alpha}^{4}=\frac{2 B_{\alpha}^{\prime}+C_{\alpha}}{q_{\alpha}+2} T_{0, \alpha}^{4} .
\end{gathered}
$$

The resulting temperature is plotted in Figures 6 and 7 Two important results are deduced for big grains from this solution: i) big grains maintain the temperature inversion and ii) if small grains set the maximum temperature limit then big grains, which are always colder than 
small grains (see equation 22), cannot reach the sublimation temperature.

However, since we do not put limits on the external flux, the solution that allows the maximum possible external flux is the one that also maximizes $F_{\text {out }}$. From equation 33 we see that the maximum $F_{\text {out }}=\sigma_{S B} T_{s u b}$ is achieved when $T_{2, \alpha}=T_{2, \beta}=T_{s u b}$. Such a solution is not possible unless $\Upsilon_{I R, \beta}=0$. Therefore, dust temperatures are maximized when all small grains sublimate away from the surface region and exist only at optical depths of $\exp \left(-\tau_{V}\right) \ll 1$. In other words, the external flux is maximized when the cloud surface "belongs" exclusively to big grains only.

\section{TRANSVERSE COOLING OF THE CLOUD SURFACE}

Solutions presented so far assume no time variability in any of the model parameters. The external flux is adjusted by hand to the value that maximizes the external flux and keeps dust temperatures below sublimation. In reality, however, this is a dynamical process where the equilibrium is established by dust moving around and sublimating whenever its temperature exceeds the sublimation point.

The existence of temperature inversion is difficult to understand under such dynamical conditions. Since the very surface of the cloud is below the sublimation point, its dust can survive closer to the external energy source than the dust within the cloud. Therefore, the distance of the cloud from the source is not defined by the very surface, but rather by the dust at the peak temperature within the cloud. Imagine now that the whole cloud is moving closer to the source. From the cloud point of reference, this dynamical process is equivalent to increasing the external flux. According to solutions in $\$ 2$ and $\$ 3$. the peak temperature within the cloud will exceed sublimation at a certain distance from the source and the dust will start to sublimate. This point is the distance at which the dust cloud seemingly stops. However, there is nothing to stop the very surface layer of the cloud to move even closer than the rest of the cloud because its temperature is below sublimation.

Notice that we can not resolve this issue in the approximation of an infinite dusty slab because the transverse optical depth (parallel to the slab surface) is always infinite. No flux can escape the slab in the transverse direction. Hence, the radiative transfer in a slab does not depend on spatial scale and only the optical depth matters. The spatial extension of the surface layer is irrelevant and solutions from $\$ 2$ and $₫ 3$ are applicable irrespectively of the dust dynamics on the spatial scale.

In reality, however, the cloud is finite and dust sublimation can eventually make the cloud optically thin in the transverse direction. This optical depth gap enables thermal radiation to flow out in the transverse direction and thus provides a channel for thermal cooling. Under such conditions the surface layer cam move closer and closer to the energy source by simultaneously expanding the size of the transverse optical depth gap, which then increases the amount of escaping thermal flux. Since the very surface dust is getting closer to the energy source, its temperature increases and eventually it reaches the dust sublimation. At that moment the radiative and dynamical equilibria are established and the whole dust cloud seemingly stops.

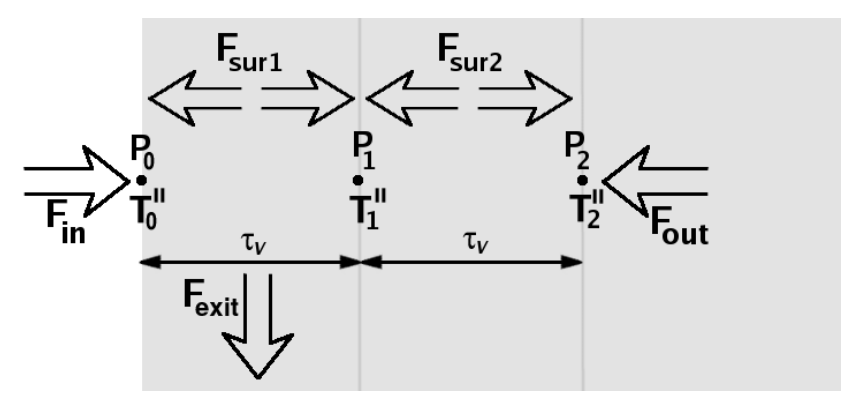

FIG. 8. - Sketch of the surface of an optically thick dust cloud illuminated from outside and cooled through the surface layer in the transverse direction. See 4 for details.

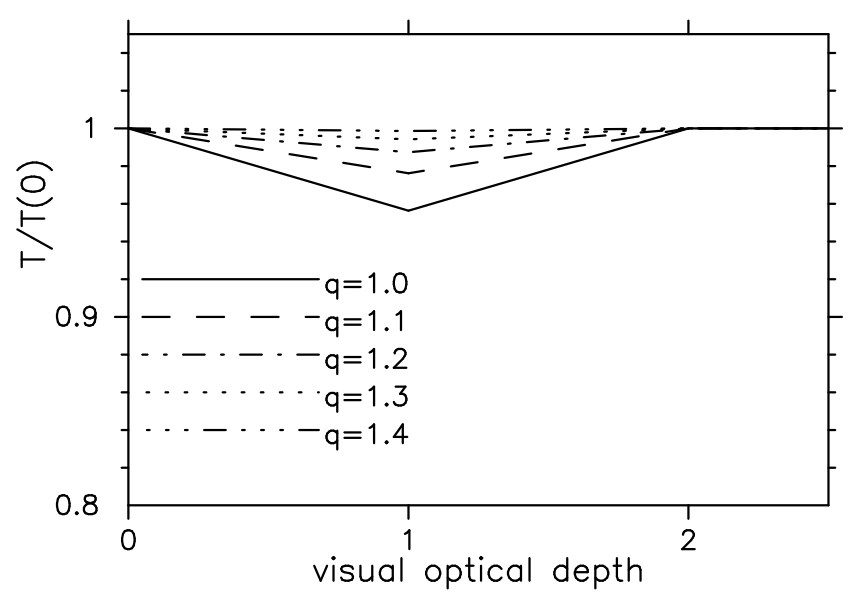

FIG. 9.- Surface temperature of an optically thick single size grain dust cloud cooled transversely through the surface layer (equations 47 and 48). The amount of transverse cooling is adjusted to the maximum temperature $T(0)$ at the very surface (equation (49). Line styles correspond to different ratios $q$ of visual to infrared opacity. Optical depth step is $\tau_{V}=1$. Increase in $T(0)$, combined with the temperature inversion (see also Fig. 2), creates a local temperature minimum within the surface layer.

The resulting dust cloud has a large surface zone where the thermal flux can transversely escape the cloud. Here we make an attempt to describe this on a quantitative level through a model shown in Figure 8. It is similar to the infinite slab model described in 92 except that now we introduce the flux $F_{\text {exit }}$ exiting the cloud transversely through the surface layer.

The flux balance equations 3 and 4 remain unchanged, while equation 2 is changed to

$$
F_{\text {in }}=F_{\text {exit }}+F_{\text {sur } 1}+F_{\text {sur } 2} e^{-\tau_{V} / q}+F_{\text {out }} e^{-2 \tau_{V} / q} .
$$

By following the same procedure as in \$2, we obtain temperatures $T_{k}^{\prime \prime}$ at points $\mathbf{P}_{k}$

$$
\begin{gathered}
F_{\text {in }}=\frac{4 \sigma_{S B}}{q+2} T_{0}^{\prime \prime 4}+\frac{2}{q+2} F_{\text {exit }} \\
T_{1}^{\prime \prime 4}=\frac{A+B}{q+2} T_{0}^{\prime \prime 4}-\left(\frac{1}{1+e^{-\tau_{V} / q}}-\frac{A+B}{2(q+2)}\right) F_{\text {exit }} \\
T_{2}^{\prime \prime 4}=\frac{2 B}{q+2} T_{0}^{\prime \prime 4}-\left(\frac{1}{1+e^{-\tau_{V} / q}}-\frac{B}{q+2}\right) F_{\text {exit }}
\end{gathered}
$$

where $\mathrm{A}$ and $\mathrm{B}$ are given in equations 15 and 16. The values in parentheses are always positive. 
We compare this with the original solution in equations 9. 13 and [14. Notice that keeping the interior temperature to be the same as before $\left(T_{2}^{\prime \prime}=T_{2}\right)$ requires a larger surface temperature $\left(T_{0}^{\prime \prime}>T_{0}\right)$. If the surface dust of $T_{0}^{\prime \prime}$ is dynamically driven toward the external energy source, as we discussed above, then it will eventually reach the sublimation temperature $T_{s u b}$. This dynamical process is accompanied by dust sublimation in the cloud interior. Sublimation creates a transverse optical depth gap and maintains $T_{2}^{\prime \prime}=T_{s u b}$. The established equilibrium condition $T_{0}^{\prime \prime}=T_{2}^{\prime \prime}=T_{\text {sub }}$ yields

$$
F_{\text {exit }}=\frac{2 B-q-2}{(q+2) /\left(1+e^{-\tau_{V} / q}\right)-B} T_{s u b}^{4} .
$$

This flux is positive for values of $q$ that produce temperature inversion $\left(q<q_{\text {limit }}\right)$. It originates from the diffuse radiation trapped within the cloud. Figure 9 shows temperature profiles for $q<q_{\text {limit }}$ based on $F_{\text {exit }}$ from equation 49. The surface layer now has a local temperature minimum at $T_{1}^{\prime \prime}$.

\section{DISCUSSION}

According to equation 21, the temperature of dust optically deep inside an optically thick cloud depends solely on the local diffuse flux. Since this can give a misleading impression that temperature effects on the cloud surface have no influence on the cloud interior, it is worth explaining why the surface solution is so important for the overall solution.

At optical depths $\tau_{V} \gtrsim 2$ the flux is exclusively diffuse and the dust temperature is dictated by equation 21. Notice, however, that even though we set the limit of zero net flux flowing through the cloud, there is no limit on the absolute value of the local diffuse flux. Arbitrarily large but equal diffuse fluxes can flow in opposite directions producing the net zero flux. It is the radiative transfer in the surface layer that sets the absolute scale. The cloud surface of $\tau_{V} \lesssim 2$ converts the directional external flux of stellar spectral shape into the diffuse thermal flux. Hence, this conversion determines the value of diffuse flux at $\tau_{V} \sim 2$, which then propagates into the cloud interior of $\tau_{V} \gtrsim 2$.

A more rigorous analytic approach shows that the net flux is not exactly zero. A very small flux, in comparison with the external flux, goes through the cloud and creates a temperature gradient. This yields the well known gray opacity solution (Mihalas 1978) where $T^{4}(\tau)=a \tau+b$. While the constant $a$ depends only on the net flux $(a=0$ in the case of zero net flux), the constant $b$ is determined by the boundary condition on the cloud surface. Hence, this becomes a reiteration of the role of the surface layer described above.

The possibility of the temperature inversion effect described in this paper was discussed previously by Wolf (2003). He noticed in his numerical calculations that larger grains can have a higher temperature than smaller grains in the surface of circumstellar dusty disks. He correctly attributed this effect to the more efficient heating of large grains by the IR radiation from the disk interior, but did not analyze it further. This is the same effect noticed by Dullemond (2002) in his numerical models of circumstellar disks with gray dust opacities. However, it was not until Isella \& Natta (2005) and Vinković et al. (2006) described this effect analytically in more detail that its importance to the overall disk structure was finally recognized.

The main driver for the detailed description of the temperature inversion effect came from the need for a better understanding of inner regions of dusty disks around young pre-main-sequence stars. The advancements of near infrared interferometry enabled direct imaging of these inner disk regions and resulted in the discovery of inner disk holes produced by dust sublimation (Monnier \& Millan-Gabet 2002). However, developing a self-consistent model that would incorporate the spectral and interferometric data proved to be a difficult problem. Two competing models are proposed. In one the data are explained by a disk that has a large vertical expansion (puffing) at the inner dust sublimation edge due to the direct stellar heating of the disk interior (Dullemond et al. 2001). In the other model the inner disk is surrounded by an optically thin dusty outflow, without a need for special distortions to the vertical disk structure (Vinković et al. 2006).

Although current observations cannot distinguish between these two models, theory gives some limits on the dust properties in the former model. The inner disk edge has to be populated by big dust grains $(\gtrsim 1 \mu \mathrm{m})$ (Isella \& Natta 2005; Vinković et al. 2006) in order to produce a large and bright vertical disk puffing needed to fit the data. The hallmark of this radiative transfer solution is the dust temperature inversion, which we also demonstrate in \$2. Vinković et al. (2006) argue that purely big grains are unrealistic because dust always comes as a mixture of grain sizes (especially in dusty disks where dust collisions should constantly keep small grains in the mix). On the other hand, Isella \& Natta (2005) point out that smaller grains should be sublimated away from the very surface of the inner disk edge, but cannot prove that this process is efficient enough to preserve dust temperature inversion.

Our analytical analysis in 33 proves that multigrain radiative transfer solutions keep small grains away from the immediate surface of optically thick disks and preserve temperature inversion. This is achieved under the boundary condition of maximum possible temperature reached by all dust grains. Then the surface becomes too hot for small grains to survive, which leaves it populated only by big grains. By choosing this favorable boundary condition, we made an important presumption about the dust dynamics. Dust has to be dynamically transported to the distances where all grains start to sublimate. This can be naturally occurring in optically thick disks due to dust and gas accretion. However, in $\$ 4$ we discovered that big grains can survive closer to the star than the inner edge of optically thick disk. The only requirement is that the disk becomes vertically optically thin at these close distances.

Therefore, the dust sublimation zone is not a simple sharp step-like transition, but rather a large zone (figure 10). We estimate its size by considering the inner radius of optically thick and thin disks (Vinković et al. 2006)

$R_{\text {in }}=\frac{\Psi R_{*}}{2}\left(\frac{T_{*}}{T_{\text {sub }}}\right)^{2}=0.0344 \Psi\left(\frac{1500 K}{T_{\text {sub }}}\right)^{2} \sqrt{\frac{L_{*}}{L_{\odot}}}[\mathrm{AU}]$

where $T_{*}, R_{*}$ and $L_{*}$ are the stellar temperature, radius and luminosity, respectively, and $\Psi$ is the the correction 


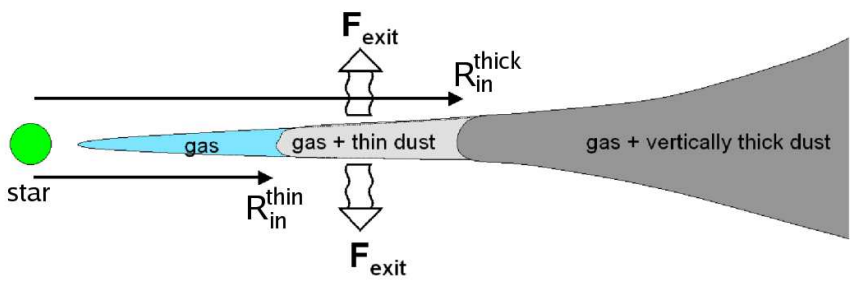

FIG. 10.- Dust dynamics in protoplanetary disks brings dust closer to the star than the inner edge of optically thick dusty disk. According to the radiative transfer solution in 44 such a disk structure is possible if this zone is populated only by big grains and it is vertically optically thin. These conditions enable disk thermal cooling and dust survival (see also figure 8). The resulting sublimation zone spans from the optically thin to the optically thick inner disk radius (equation 50). Its physical size is $\sim 0.2 \mathrm{AU}$ in Herbig Ae stars and $\sim 0.03 \mathrm{AU}$ in $\mathrm{T}$ Tau stars.

for diffuse heating from the disk edge interior. Optically thick disks have $\Psi=2$, while optically thin disks have $\Psi \sim 1.2$. The sublimation zone exists between these two extremes, which translates to $\sim 0.2 \mathrm{AU}$ for Herbig Ae stars $\left(\sim 50 L_{\odot}\right)$ or $\sim 0.03 \mathrm{AU}$ in $\mathrm{T}$ Tau stars $\left(\sim L_{\odot}\right)$. This is a considerable size detectable by interferometric imaging and has to be addressed in future studies. Also, its gas is enriched by metals coming from the sublimated dust, which makes this zone a perfect place for dust growth. Notice that, in addition to sublimation, grain growth is another way of making the disk optically thinner. Hence, the evolutionary role of such a large sublimation zone has to be studied further in more detail.

Another major problem with the dynamics of big grains is that they tend to settle toward the midplane, which suppresses vertical expansion of the inner disk edge and leads to the failure of the disk puffing model (Dullemond \& Dominik 2004; Vinković et al. 2006). On the other hand, the concurrent model incorporating a dusty outflow lacks a convincing physical process responsible for the formation of a dusty wind. The sublimation zone may play an important role in dusty wind processes, since its small optical depth and small distance from the star should also result in gas properties that are more susceptible to non-gravitational forces capable of launching a dusty wind (such as magnetic fields) than the rest of the dusty disk.

\section{CONCLUSION}

We analyzed the temperature structure of externally heated optically thick dust clouds. We focused on the recently discovered effect of temperature inversion within the optically thin surface of a cloud populated by big $(\geq$ $1 \mu \mathrm{m}$ ) dust grains (Isella \& Natta 2005; Vinković et al. 2006). The effect is produced by reprocessing the external radiation and not by any additional energy source.
The inversion manifests itself as a temperature increase with optical depth, before it starts to decrease once the external directional radiation is completely transformed into the diffuse thermal flux. Since small grains do not show this effect, the open question was whether small grains would manage to suppress this effect when mixed with big grains.

We show analytically that small grains remove the temperature inversion of big grains if the overall opacity is dominated by small grains. However, this does not happen in situations where the cloud is close enough to the external energy source for dust to start sublimating. Small grains acquire a higher temperature than bigger grains and sublimate away from the immediate cloud surface. The exact grain size composition of the surface depends on the amount of external flux because different grain sizes sublimate at different distances from the surface. These distances are smaller than in optically thin clouds because bigger grains provide shielding to smaller grains from direct external radiation. We show that the temperature inversion is always preserved if small grains are removed from only $\tau_{V} \sim 1$ of the immediate surface.

If the boundary condition requires all dust sizes to maximize their temperature, then all small grains are removed from the surface layer. They can exist only within the cloud interior $\exp \left(-\tau_{V}\right) \ll 1$ where the external radiation is completely absorbed. Such a condition is expected in protoplanetary disks where dust accretion moves dust toward the star. The inner disk radius is then defined by the largest grains, no matter what the overall grain size composition, because the largest grains survive the closest to the star and dictate the surface radiative transfer.

A new problem arises in that case. Since the temperature inversion keeps the very surface of the cloud below the sublimation temperature, its dust can move even closer to the star. We show that this creates an optically thin dusty zone inside the dust destruction radius of an optically thick disk (figure 10). Only big grains can survive in this zone. We estimate that its size is large enough to be detected by near IR interferometry. It consists of big grains and gas enriched by metals from sublimated dust, hence favorable for grain growth. This shows that the geometry and structure of inner disks cannot be determined by simple ad hoc boundary conditions. It requires self-consistent calculations of dust dynamics combined with radiative transfer calculations and dust sublimation.

Supports by the NSF grant PHY-0503584 and the W.M. Keck Foundation are gratefully acknowledged.

\section{REFERENCES}

Calvet, N., Patino, A., Magris, G. C., \& D'Alessio, P. 1991, ApJ, 380,617

Carciofi, A. C., Bjorkman, J. E., \& Magalhães, A. M. 2004, ApJ, 604, 238

Dullemond, C. P. 2002, A\&A, 395, 853

Dullemond, C. P., Dominik, C., \& Natta, A. 2001, ApJ, 560, 957

Dullemond, C. P., \& Dominik, C. 2004, A\&A, 417, 159

Efstathiou, A., \& Rowan-Robinson, M. 1994, MNRAS, 266, 212

Isella, A., \& Natta, A. 2005, A\&A, 438, 899
Malbet, F., \& Bertout, C. 1991, ApJ, 383, 814

Mihalas, D. "Stellar atmospheres", San Francisco, W. H. Freeman and Co., 1978.

Monnier,J. D. \& Millan-Gabet, R. 2002, ApJ579, 694

Vinković, D., Ivezić, Ž., Jurkić, T., \& Elitzur, M. 2006, ApJ, 636, 348

Wolf, S. 2003, ApJ, 582, 859 